\title{
Special issue from the 12th North American Forest Ecology Workshop
} (NAFEW 2019)

\author{
Guest Editors \\ Kristen Waring, School of Forestry, Northern Arizona University, Flagstaff, Arizona, USA \\ John Bradford, U.S. Geological Survey, Southwest Biological Science Center, Flagstaff, Arizona, USA \\ Christopher Looney, Pacific Southwest Research Station, USDA Forest Service, Davis, California, USA
}

